\title{
Scaling and memory in the return intervals of realized volatility
}

\author{
Fei Ren ${ }^{\mathrm{a}, \mathrm{b}, \mathrm{c}}$, Gao-Feng Gu ${ }^{\mathrm{a}, \mathrm{b}, \mathrm{d}}$, Wei-Xing Zhou ${ }^{*, \mathrm{a}, \mathrm{b}, \mathrm{c}, \mathrm{d}, \mathrm{e}}$ \\ ${ }^{a}$ School of Business, East China University of Science and Technology, Shanghai 200237, China \\ ${ }^{b}$ Research Center for Econophysics, East China University of Science and Technology, Shanghai 200237, China \\ ${ }^{c}$ Engineering Research Center of Process Systems Engineering (Ministry of Education), East China University of Science and Technology, \\ Shanghai 200237, China \\ ${ }^{d}$ School of Science, East China University of Science and Technology, Shanghai 200237, China \\ ${ }^{e}$ Research Center on Fictitious Economics \& Data Science, Chinese Academy of Sciences, Beijing 100080, China
}

\begin{abstract}
We perform return interval analysis of 1-min realized volatility defined by the sum of absolute high-frequency intraday returns for the Shanghai Stock Exchange Composite Index (SSEC) and 22 constituent stocks of SSEC. The scaling behavior and memory effect of the return intervals between successive realized volatilities above a certain threshold $q$ are carefully investigated. In comparison with the volatility defined by the closest tick prices to the minute marks, the return interval distribution for the realized volatility shows a better scaling behavior since 20 stocks (out of 22 stocks) and the SSEC pass the Kolmogorov-Smirnov (KS) test and exhibit scaling behaviors, among which the scaling function for 8 stocks could be approximated well by a stretched exponential distribution revealed by the KS goodnessof-fit test under the significance level of 5\%. The improved scaling behavior is further confirmed by the relation between the fitted exponent $\gamma$ and the threshold $q$. In addition, the similarity of the return interval distributions for different stocks is also observed for the realized volatility. The investigation of the conditional probability distribution and the detrended fluctuation analysis (DFA) show that both short-term and long-term memory exists in the return intervals of realized volatility.
\end{abstract}

Key words: Econophysics; Realized volatility; Return interval; Scaling; Long memory

PACS: 89.65.Gh, 89.75.Da, 05.45.Tp

\section{Introduction}

The study of extreme events has drawn much attention of scientists, for instance the nature records of floods, temperatures and earthquakes [1, 2, 3, 4, 5, 6]. By investigating the return intervals between successive extreme events exceeding a certain threshold $q$, scaling behaviors are revealed in the return interval distributions for numerous complex systems $[3,4,5,6]$. As with this scaling behavior, we can infer that the probability distribution of the return intervals of rare events that are difficult to take empirical measurements. This scaling property of extreme events is supposed to be of great importance for the risk assessment of rare events. Further studies have shown that the scaling behavior of return intervals may arise from the long-term memory of the original records [7, 8, 9, 10, 11]. This suggests that the scaling behavior might also appear in other types of records with long-term correlations, such as the stock market records.

The early study of extreme events in stock markets mainly concerns the return intervals between successive volatilities above a certain threshold $q$. The time intervals between consecutive trades and orders have also been widely studied [12, 13, 14, 15, 16, 17]. In general, the volatility $R(t, \delta t)$ is simply defined as the magnitude of logarithmic return between tick prices at time $t$ and $t-\delta t$, i.e. $R(t, \delta t)=|\ln (Y(t))-\ln (Y(t-\delta t))|$, where $Y(t)$ is the tick price at time $t$. With this definition, Yamasaki et al and Wang et al used the daily data and intraday data of US stocks to

${ }^{*}$ Corresponding author. Address: 130 Meilong Road, P.O. Box 114, School of Business, East China University of Science and Technology, Shanghai 200237, China, Phone: +8621 64253634, Fax: +862164253152.

Email address: wxzhou@ecust . edu. cn (Wei-Xing Zhou)

Preprint submitted to Physica A

October 30, 2018 
study the probability distribution of volatility return intervals, and indeed found a scaling behavior [18, 19, 20, 21]. Further studies show that long-term memory also exists in the volatility return intervals. Similar scaling behavior and long-term memory are observed in thousands Japanese stocks and 4 Chinese stocks [22, 23]. On the contrary, there is growing evidence showing that the return interval distribution may exhibit multiscaling behavior. Lee et al studied the 1-min volatility data of the Korean KOSPI index [24], and Wang et al analyzed the trade-by-trade data of 500 stocks composing the S\&P 500 index and 1137 US common stocks [25, 26], and Kaizoji analyzed the daily data of 800 companies listed on Tokyo Stock Exchange and the Nikkei 225 index [27], and Ren and Zhou studied two Chinese stock market indexes [28], and all these studies show that the return interval distributions for different thresholds $q$ exhibit a systematic deviation from scaling and show multiscaling behavior. Ren, Guo and Zhou conducted a more careful study to scrutinize 30 very liquid stocks in the Chinese market using the Kolmogorov-Smirnov (KS) test, and found that some stocks pass the KS test displaying scaling behaviors while others show multiscaling behaviors [29].

In the finance literature, there are many different estimators for volatility. Anderson et al proposed a daily realized volatility constructed from the sum of the square intraday returns [30, 31]. This realized daily volatility contains more valuable information about the intraday data, and is made arbitrarily close to the underlying integrated volatility. In addition, there are other daily volatility estimators constructed based on the intraday data [32, 33, 34, 35]. Subsequently, this realized volatility is generalized to the volatility which sums the square returns in a fixed time interval [36]. This realized volatility may better describe the price fluctuation caused by the trades occurring in that time interval. It is interesting to investigate the statistical properties of return intervals of realized volatility.

In this paper, we investigate the statistical properties of the return intervals of realized volatility based on highfrequency intraday data in the Chinese stock market. Inspired by the sum of squared returns realized volatility raised by Anderson, we introduce an estimate of 1-min realized volatility by summing the absolute logarithmic returns utilizing all trading data in each minute. This 1-min realized volatility contains more valuable information which lies in the high frequency trading data. Using the Kolmogorov-Smirnov (KS) test and detrended fluctuation analysis (DFA) method, we test if the scaling behavior and long-term memory of the return intervals maintain with this 1-min realized volatility.

The paper proceeds as follows: In Section 2 we introduce the database analyzed and the definition of 1-min realized volatility. In Section 3, we study the return interval distribution of the realized volatility using the KS tests. In Section 4 , we further study the memory effect of the realized volatility return intervals. Section 5 summarizes.

\section{Volatility definition}

Our analysis is based on the high-frequency intraday data of SSEC and 22 liquid stocks traded on the Shanghai Stock Exchange. These 22 stocks are the most actively traded stocks representative of a variety of industry sectors, and consequently have the largest sizes among all the stocks. The prices and the associated times of the SSEC index and the individual stocks are recorded every six to eight seconds from January 2004 to June 2006.

In many previous studies, the volatility is defined as the magnitude of the logarithmic return,

$$
R_{1}(t)=|\ln Y(t)-\ln Y(t-1)|,
$$

where $\mathrm{Y}(\mathrm{t})$ is the closest tick price to a minute mark $t[19,20,21,24,28,29]$. In this paper, we focus on the realized volatility constructed from the sum of absolute trade-by-trade returns within one minute. Suppose that $Y\left(t^{\prime}\right)$ is the tick price at time $t^{\prime}$, then the realized volatility is defined as

$$
R_{2}(t)=\sum_{t-1<t^{\prime} \leqslant t}\left|\ln Y\left(t^{\prime}\right)-\ln Y\left(t^{\prime}-1\right)\right|,
$$

where the sum is taken over all the tick times between $t-1$ and $t$. For both volatility definitions the sampling time is one minute, and the volatility data size is about 140,000 for each stock.

Before doing the analysis, we removed the intraday pattern to eliminate its periodic effect on the return interval distribution [37, 29] via dividing the volatility $R_{i}(t)$ by its average value corresponding to time $t$ on the day [19, 20, 21]. Then we normalize the data by dividing its standard deviation so that the volatility is in units of its standard deviation. 


\section{Probability distribution of realized volatility return intervals}

\subsection{Empirical return interval distribution}

We study the return intervals $\tau$ between successive volatilities exceeding a certain threshold $q$. For each threshold $q$, a series of return intervals is obtained and its empirical probability distribution $P_{q}(\tau)$ can be obtained. Many empirical studies have showed that the probability distribution function (PDF) of the scaled return intervals may obey a scaling form

$$
P_{q}(\tau)=\frac{1}{\langle\tau\rangle} f(\tau /\langle\tau\rangle)
$$

where $\langle\tau\rangle$ is the mean return interval which depends on the threshold $q$.

Ren et al have studied the return intervals of the volatility defined by $R_{1}$ for the SSEC and the 22 constituent stocks analyzed in this paper [28, 29]. They found that, for some of the stocks and the SSEC, the return interval distributions do not show scaling behavior. In Fig. 1 (a) and (b), the PDFs of the scaled return intervals of volatility $R_{1}$ for the SSEC and a representative stock 600028 are plotted. For the SSEC, the curves for different thresholds $q=2,3,4,5$ do not collapse to a single curve and show systematic deviation from scaling. For stock 600028 , the deviation becomes relatively small, but one can still see some difference between the curves. Fig. 1 (c) and (d) plot the return interval PDFs of the realized volatility $R_{2}$ for the SSEC and stock 600028 for comparison. We find that the curves for different $q$ values approximately collapse to a single solid curve. This strongly suggests that the return interval distribution for the realized volatility shows better scaling behavior than that of the volatility defined by $R_{1}$.
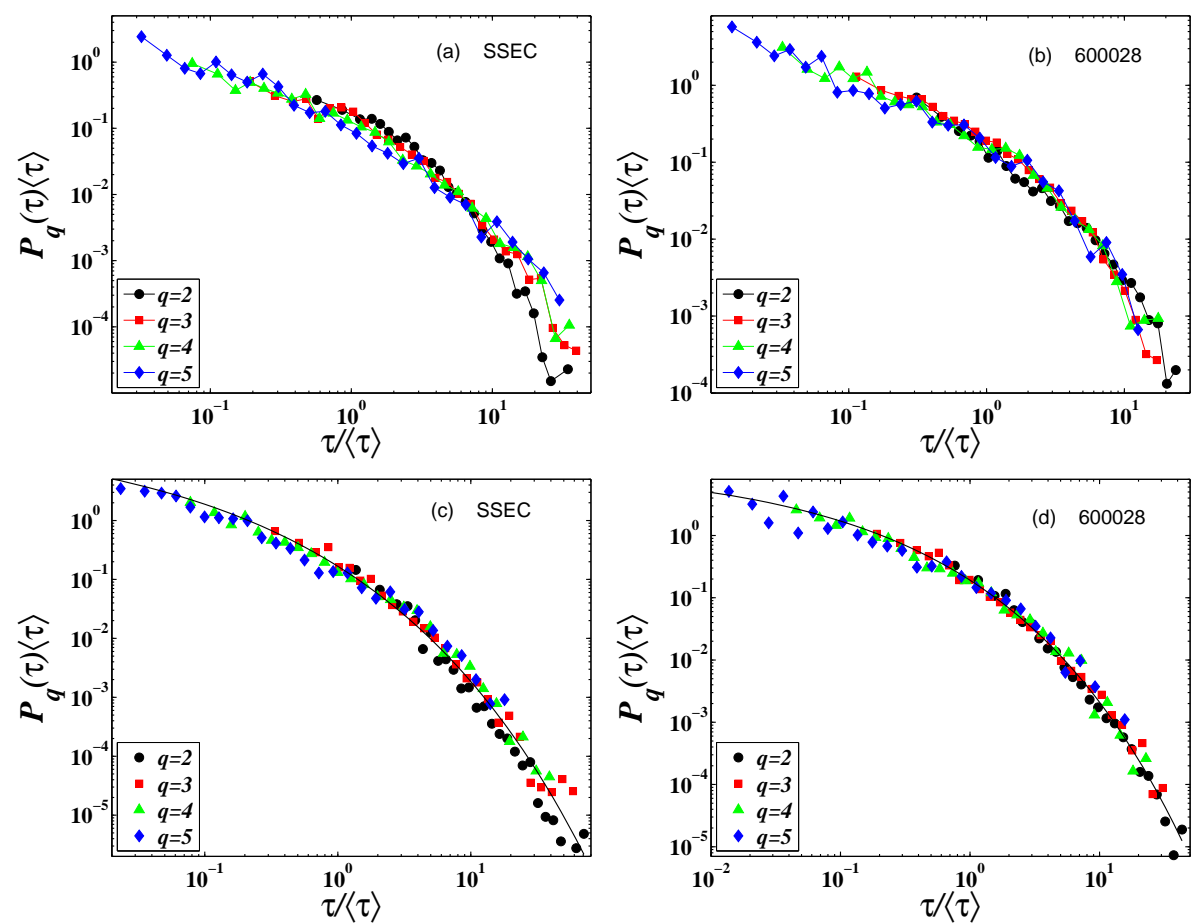

Figure 1: (Color online) Probability distributions of the scaled return intervals of volatilities defined by (a) $R_{1}$ for SSEC, (b) $R_{1}$ for stock 600028 , (c) $R_{2}$ for SSEC, and (d) $R_{2}$ for stock 600028. Solid curves in (c) and (d) are stretched exponential fits with $\gamma=0.26$ and 0.31 respectively.

\subsection{Kolmogorov-Smirnov test of scaling in $P_{q}(\tau)$ for different $q$}

Eyeballing of the return interval distributions offers a qualitative way of distinguishing the scaling and nonscaling behavior. A quantitative method, the Kolmogorov-Smirnov (KS) test, is further adopted to examine the possible collapse of the interval distributions for different thresholds. We use the KS test to examine two return interval distributions for $q=2$ and 5, which seems to behave most differently among all the distributions. If the distributions 
for $q=2$ and 5 pass the test, we can conclude that all the return interval distributions for different $q$ values collapse onto a single curve and consequently obey a scaling law. Suppose that $F_{2}$ is the cumulative distribution function (CDF) of return intervals for $q=2$ and $F_{5}$ is the CDF of return intervals for $q=5$. We calculate the $K S$ statistic by comparing the two CDFs in the overlapping region:

$$
K S=\max \left(\left|F_{2}-F_{5}\right|\right) .
$$

When the $K S$ statistic is smaller than a critical value denoted by $C V$ (i.e., $K S<C V$ ), we can conclude that the distribution for $q=2$ is coincident with the distribution for $q=5$, and the return interval distributions show scaling behavior. The critical value is $C V=c_{\alpha} / \sqrt{m n /(m+n)}$, where $m$ and $n$ are the numbers of interval samples for $q_{i}$ and $q_{j}$ [38, 39], and the threshold is $c_{\alpha}=1.36$ at the significance level of $\alpha=5 \%$ [40, 41].

Ren, Guo and Zhou have used the KS test to examine the scaling behavior of the return interval distribution for the volatility defined by $R_{1}$, and found that among the 22 chosen stocks, 11 of the individual stocks pass the KS test and show scaling behavior, while the remaining 11 stocks as well as the SSEC do not show scaling behavior as depicted in Table 1. We also use the KS test to study the scaling behavior of the return interval distribution for the realized volatility defined by $R_{2}$. Remarkably, only 2 stocks fail in the test, and the remaining 20 stocks pass the test and show scaling behavior. More interestingly, the SSEC also exhibits scaling behavior, as manifested by the collapse of the distributions in Fig. 1 This confirms that the scaling behavior of the return interval distribution is significantly improved when the realized volatility $R_{2}$ is adopted. The improved scaling behavior of the realized volatility return interval distribution is consistent with the scaling behavior of the return interval distribution for 4 Chinese stocks reported by Qiu et al [23]

\subsection{Function form of $P_{q}(\tau)$}

Ren, Guo and Zhou have further performed the KS goodness-of-fit test [42, 43] to study the particular form of the scaling function using the volatility defined by $R_{1}$, and confirmed that the scaling function of the stocks which show good scaling behavior could be approximated by a stretched exponential form

$$
f(\tau /\langle\tau\rangle)=c e^{-a(\tau /\langle\tau\rangle)^{\gamma}} .
$$

We adopt the same method to test the hypothesis that the empirical distributions for different $q$ values for the realized volatility defined by $R_{2}$ are coincident with a single stretched exponential fit. Following Ren et al [29], we test the return interval distributions for two special $q$ values $q=2$ and 5 which behave most differently among all the distributions. Only the 20 stocks which show scaling behaviors and the SSEC are tested. If both return interval distributions for $q=2$ and 5 are identical to a same stretched exponential in the overlapping region of the scaled return intervals, we can conclude that the scaling function has a stretched exponential form.

In the case of the KS goodness-of-fit test, the $K S$ statistic calculates the difference between the cumulative distribution $F_{q}$ of empirical return intervals and the cumulative distribution $F_{\mathrm{SE}}$ from the fitted stretched exponential,

$$
K S=\max \left(\left|F_{q}-F_{\mathrm{SE}}\right|\right), \quad q=2,5 .
$$

A weighted $K S$ statistic, which is more sensitive on the edges of the cumulative distribution, is defined as [43]

$$
K S W=\max \left(\frac{\left|F_{q}-F_{\mathrm{SE}}\right|}{\sqrt{F_{\mathrm{SE}}\left(1-F_{\mathrm{SE}}\right)}}\right) .
$$

We generate 1000 synthetic samples from the best fitting distribution, and calculate the $K S$ and $K S W$ statistics for the synthetic data by taking the same measurements as we do for the empirical data as

$$
K S_{\text {sim }}=\max \left(\left|F_{\text {sim }}-F_{\text {sim,SE }}\right|\right)
$$

\footnotetext{
${ }^{1}$ We have discussed with Qiu and revealed that they defined the volatility in a similar way as we did for the realized volatility $R_{2}$. However, they did not clearly clarify this in their paper [23].
} 
Table 1: Kolmogorov-Smirnov test of the return interval distributions by comparing the statistic $K S$ with the critical value $C V$ at the $5 \%$ significance level for SSEC and 22 constituent stocks.

\begin{tabular}{cccccccc}
\hline \multirow{2}{*}{ Stock code } & \multicolumn{3}{c}{ Volatility $R_{1}$} & & \multicolumn{3}{c}{ Volatility $R_{2}$} \\
\cline { 2 - 4 } \cline { 6 - 7 } & $K S$ & $C V$ & Scaling? & & $C S$ & $C V$ & Scaling? \\
\hline SSEC & 0.1170 & 0.0434 & No & & 0.0455 & 0.0502 & Yes \\
600000 & 0.0699 & 0.0538 & No & & 0.0243 & 0.0481 & Yes \\
600019 & 0.0223 & 0.0672 & Yes & & 0.0407 & 0.0701 & Yes \\
600026 & 0.0277 & 0.0511 & Yes & & 0.0362 & 0.0516 & Yes \\
600028 & 0.0670 & 0.0653 & No & & 0.0374 & 0.0652 & Yes \\
600030 & 0.0580 & 0.0520 & No & & 0.0409 & 0.0492 & Yes \\
600036 & 0.0436 & 0.0576 & Yes & & 0.0466 & 0.0535 & Yes \\
600073 & 0.0501 & 0.0500 & No & & 0.0345 & 0.0452 & Yes \\
600088 & 0.0540 & 0.0497 & No & & 0.0250 & 0.0456 & Yes \\
600100 & 0.0462 & 0.0500 & Yes & & 0.0239 & 0.0461 & Yes \\
600104 & 0.0149 & 0.0573 & Yes & & 0.0365 & 0.0510 & Yes \\
600110 & 0.0911 & 0.0506 & No & & 0.0958 & 0.0457 & No \\
600171 & 0.0377 & 0.0530 & Yes & & 0.0204 & 0.0482 & Yes \\
600320 & 0.0515 & 0.0506 & No & & 0.0305 & 0.0469 & Yes \\
600428 & 0.0475 & 0.0510 & Yes & & 0.0345 & 0.0501 & Yes \\
600550 & 0.0198 & 0.0498 & Yes & & 0.0198 & 0.0467 & Yes \\
600601 & 0.0218 & 0.0637 & Yes & & 0.0460 & 0.0569 & Yes \\
600602 & 0.0167 & 0.0561 & Yes & & 0.0316 & 0.0513 & Yes \\
600688 & 0.0421 & 0.0566 & Yes & & 0.0391 & 0.0531 & Yes \\
600770 & 0.0647 & 0.0478 & No & & 0.0284 & 0.0442 & Yes \\
600797 & 0.1179 & 0.0647 & No & & 0.1220 & 0.0568 & No \\
600832 & 0.0610 & 0.0474 & No & & 0.0203 & 0.0469 & Yes \\
600900 & 0.0574 & 0.0556 & No & & 0.0300 & 0.0550 & Yes \\
\hline \hline
\end{tabular}


and

$$
K S W_{\text {sim }}=\max \left(\frac{\left|F_{\text {sim }}-F_{\text {sim,SE }}\right|}{\sqrt{F_{\text {sim }, \mathrm{SE}}\left(1-F_{\text {sim }, \mathrm{SE})}\right.}}\right),
$$

where $F_{\text {sim }}$ is the CDF of each simulated synthetic sample and $F_{\text {sim,SE }}$ is the CDF of its best fit obtained from integrating the fitted stretched exponential. The $p$-value is defined as the frequency that $K S_{\text {sim }}>K S$ or $K S W_{\text {sim }}>K S W$, and it can be regarded as the probability that the empirical distribution is consistent with its best fit. The tests are carried out for SSEC and 20 constituent stocks, and the resultant $p$-values are listed in Table 2

Table 2: $K S$ and $K S W$ goodness-of-fit tests of the scaling function form of return interval distributions for $q=2$ and 5 by comparing empirical data with the best stretched exponential fit and synthetic data with the best stretched exponential fit. The stocks marked with $\star$ pass the test using the $K S$ statistic, and the stocks marked with $\star \star$ pass the test using both $K S$ and $K S W$ statistics under the significant level of $5 \%$.

\begin{tabular}{lccc|lccc|lccc}
\hline \hline Code & $q$ & $p_{K S}$ & $p_{K S W}$ & Code & $q$ & $p_{K S}$ & $p_{K S W}$ & Code & $q$ & $p_{K S}$ & $p_{K S W}$ \\
\hline SSEC $^{\star}$ & 2 & 0.321 & 0.159 & $600000^{\star \star}$ & 2 & 0.338 & 0.277 & $600019^{\star}$ & 2 & 0.074 & 0.042 \\
& 5 & 0.203 & 0.002 & & 5 & 0.434 & 0.213 & & 5 & 0.530 & 0.324 \\
\hline 600028 & 2 & 0.011 & 0.001 & 600030 & 2 & 0.168 & 0.094 & $600026^{\star \star}$ & 2 & 0.630 & 0.406 \\
& 5 & 0.712 & 0.841 & & 5 & 0.018 & 0.005 & & 5 & 0.288 & 0.288 \\
\hline $600036^{\star \star}$ & 2 & 0.707 & 0.635 & $600073^{\star \star}$ & 2 & 0.417 & 0.274 & $600088^{\star \star}$ & 2 & 0.443 & 0.120 \\
& 5 & 0.209 & 0.205 & & 5 & 0.411 & 0.352 & & 5 & 0.066 & 0.056 \\
\hline 600104 & 2 & 0.013 & 0.006 & $600100^{\star \star}$ & 2 & 0.379 & 0.236 & 600171 & 2 & 0.016 & 0.001 \\
& 5 & 0.045 & 0.017 & & 5 & 0.872 & 0.665 & & 5 & 0.005 & 0 \\
\hline $600320^{\star \star}$ & 2 & 0.744 & 0.526 & 600428 & 2 & 0.010 & 0.006 & $600550^{\star}$ & 2 & 0.070 & 0.042 \\
& 5 & 0.110 & 0.051 & & 5 & 0 & 0.001 & & 5 & 0.564 & 0.531 \\
\hline $600601^{\star \star}$ & 2 & 0.493 & 0.351 & $600602^{\star}$ & 2 & 0.522 & 0.361 & 600688 & 2 & 0.037 & 0.001 \\
& 5 & 0.417 & 0.149 & & 5 & 0.130 & 0.039 & & 5 & 0.007 & 0.002 \\
\hline $600770^{\star}$ & 2 & 0.680 & 0.441 & $600832^{\star}$ & 2 & 0.532 & 0.246 & $600900^{\star}$ & 2 & 0.307 & 0.256 \\
& 5 & 0.067 & 0.015 & & 5 & 0.122 & 0.006 & & 5 & 0.122 & 0.005 \\
\hline \hline
\end{tabular}

By checking the $p$-values for both distributions for $q=2$ and 5 , we can test the null hypothesis that the empirical PDFs can be fitted well by a stretched exponential. Consider the significance level of $1 \%$, if at least one $p$-value for $q=2$ or 5 of an individual stock is less than $1 \%$, then the null hypothesis is rejected. Table 2 depicts the $p$-values for both distributions for $q=2$ and 5 for the volatility defined by $R_{2}$. According to Table 2, 17 stocks as well as the SSEC pass the goodness-of-fit test using the $K S$ statistic, and 12 stocks pass the goodness-of-fit test using the $K S W$ statistic. For the volatility defined by $R_{1}$, only 8 stocks (out of the 20 constituent stocks examined by the KS goodness-of-fit test) pass the goodness-of-fit test using the $K S$ statistic and 7 stocks pass the goodness-of-fit test using the $K S W$ statistic [29]. Consider the significance level of 5\%, 14 stocks as well as the SSEC pass the goodness-of-fit test using the $K S$ statistic, and 8 stocks pass the goodness-of-fit test using the $K S W$ statistic for the volatility defined by $R_{2}$. In contrast, for the volatility defined by $R_{1}, 6$ stocks pass the goodness-of-fit test using the $K S$ statistic and 5 stocks pass the goodness-of-fit test using the $K S W$ statistic [29]. We find that the $K S$ and $K S W$ statistics provide very similar results for the volatility defined by $R_{1}$, but the $K S W$ statistic is more sensitive than the $K S$ statistic for the volatility defined by $R_{2}$. In principle, the $p$-values of a stock are larger when the scaling of PDFs for different $q$ is more significant.

\subsection{Dependence of $\gamma$ on $q$}

It has been shown that for the stocks which show multiscaling behaviors, e.g. SSEC, the PDFs could also be well approximated by the stretched exponential distribution but with different exponent $\gamma$ for different threshold $q$ [28]. Therefore, we assume that the PDFs for the constituent stocks could be approximated by the stretched exponential function, whether they show good scaling behaviors or not. We fit the PDFs for different threshold $q$ using a stretched exponential form

$$
P_{q}(\tau)=b e^{-a(\tau /\langle\tau\rangle)^{\gamma}} .
$$


where $a, b$ and $\gamma$ are dependent of $q$ if there is no scaling. We investigate the relationship between the exponent $\gamma$ and the threshold $q$ to further study the tendency of return interval distribution with $q$.

In Fig. 2 the exponents $\gamma$ are plotted as a function of the threshold $q$ for SSEC and three representative stocks 60000,600028 and 600030 . Though the curve for the volatility defined by $R_{1}$ fluctuates a little which may due to the fitting errors caused by fluctuations, it shows an approximate decreasing tendency with an increase of $q$. Regardless of the fitting errors, the fitted exponent $\gamma$ mainly dominates the shape of the return interval distribution. The decreasing tendency of $\gamma$ further confirms our previous findings that the return interval distributions for different $q$ values distinctly differ from each other and show systematic deviation from scaling [29]. Whereas for the volatility defined by $R_{2}, \gamma$ slightly fluctuates but stays relatively constant in comparison with that of the volatility defined by $R_{1}$. A similar phenomenon is observed for other constituent stocks. This provides additional evidence that the scaling behavior of the return interval distribution for the realized volatility defined by $R_{2}$ is significantly improved.
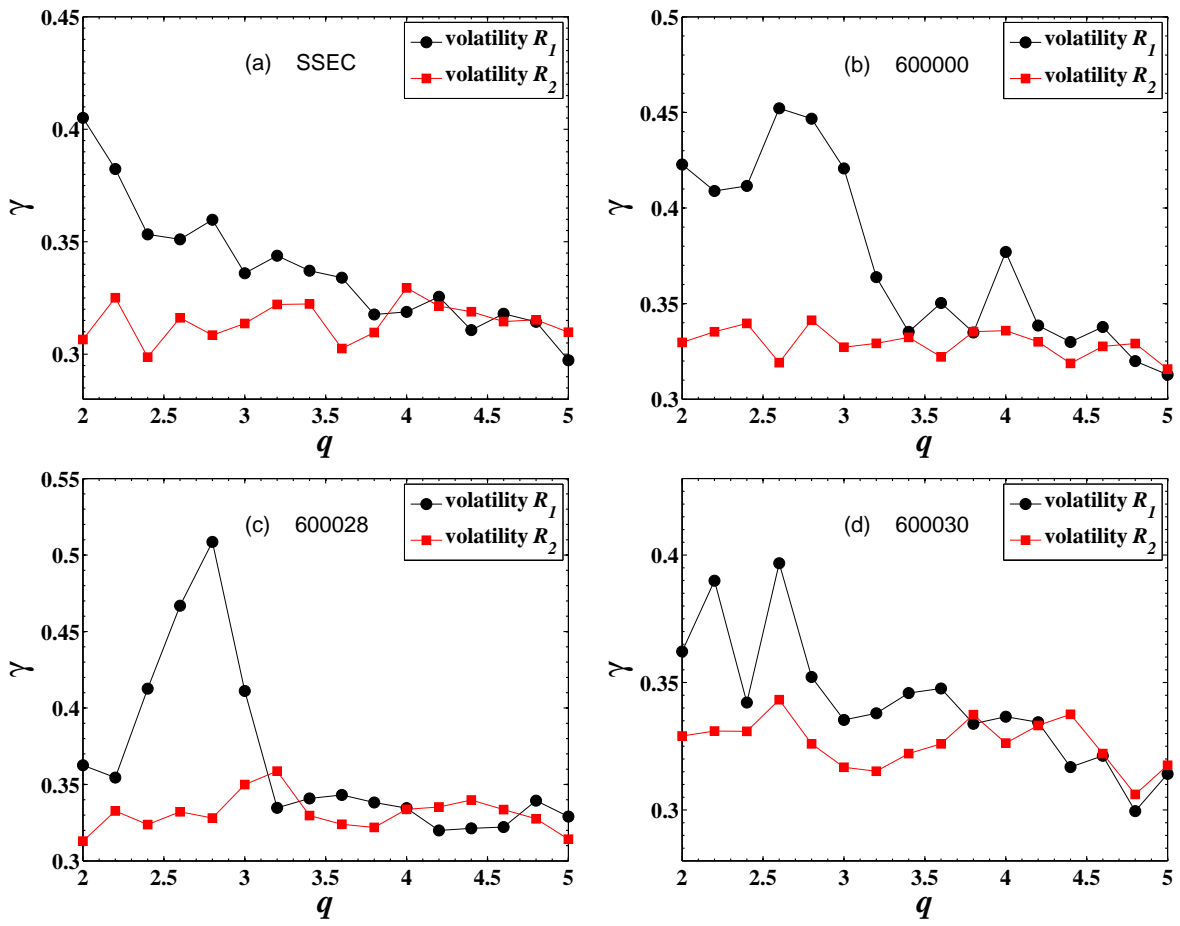

Figure 2: (Color online) Exponent $\gamma$ vs. threshold $q$ for (a) SSEC, (b) stock 600000, (c) stock 600028, and (d) stock 600030.

\subsection{Similarity of $P_{q}(\tau)$ for different stocks}

We also study the similarity of the return interval distributions for different stocks. We fix the threshold $q=2$, and see how the return interval distributions behave for different stocks. In Fig. 3 (a), the PDFs of the scaled return intervals of the volatility defined by $R_{1}$ for SSEC and five representative stocks are plotted. It is seen that the curves for different stocks differ from each other and do not collapse to a single curve. We then use the volatility defined by $R_{2}$ to investigate the PDFs of the scaled return intervals for the SSEC and the 5 representative stocks. As shown in Fig. 3 (b), the curves for different stocks have very similar shapes and seem to collapse on a single curve. This indicates that the PDFs for different stocks may follow similar scaling function if we use the volatility defined by $R_{2}$.

\section{Memory in the return intervals of realized volatility}

\subsection{Short memory in realized volatility return intervals}

The memory effect is another important characteristic feature of the stock markets. Empirical study has revealed that the memory effect universally exists in the return intervals of various stock markets for the volatility defined by 

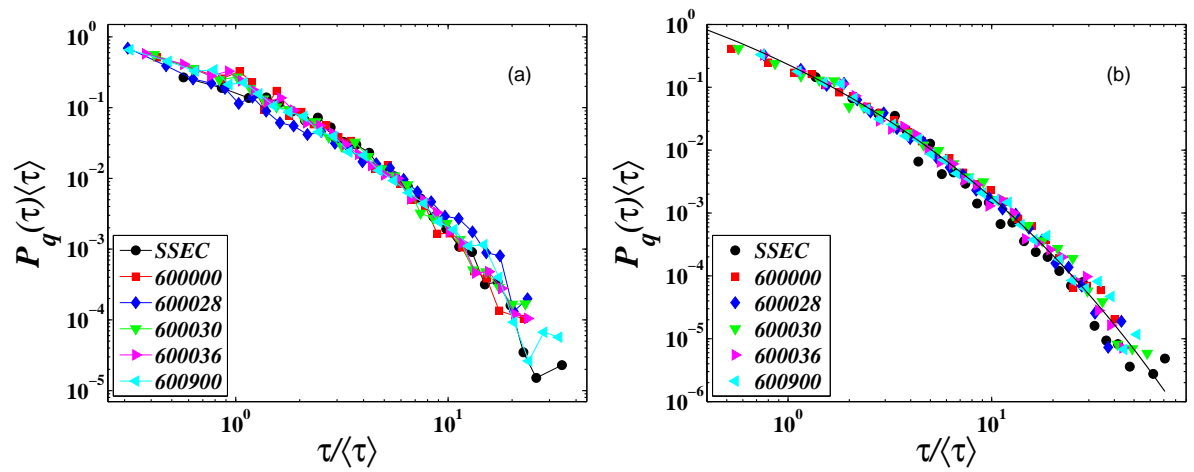

Figure 3: (Color online) Probability distributions of the scaled return intervals for SSEC and five representative constituent stocks when $q=2$ for volatilities defined by (a) $R_{1}$ and (b) $R_{2}$. The solid line in (b) is the stretched exponential fit with $\gamma=0.26$.

$R_{1}[19,20,22,23,29]$. We try to test if the memory effect retains in the return intervals when we use the realized volatility defined by $R_{2}$.

We first investigate the short-term memory of the return intervals by calculating the conditional PDF $P_{q}\left(\tau \mid \tau_{0}\right)$, defined as the probability to find an interval $\tau$ immediately after an interval $\tau_{0}$. To get better statistics, we study $P_{q}\left(\tau \mid \tau_{0}\right)$ for a bin of $\tau_{0}$. The entire interval sequences are arranged in an ascending order and partitioned into four bins with equal size. $P_{q}\left(\tau \mid \tau_{0}\right)$ for $\tau_{0}$ in the smallest and biggest quarter bins for SSEC and stock 600028 with volatility defined by $R_{1}$ are illustrated in Fig. 4 (a) and (b). The curves intersperse with each other for small scaled return intervals, and it is hard to distinguish the curve for $\tau_{0}$ in the smallest subset from that for $\tau_{0}$ in the biggest subset. In comparison with that of the volatility defined by $R_{1}, P_{q}\left(\tau \mid \tau_{0}\right)$ for the same stock index and the individual stock for the volatility defined by $R_{2}$ are plotted in Fig. 4 (c) and (d). One observes that the scaling behavior of $P_{q}\left(\tau \mid \tau_{0}\right)$ is significantly improved for the volatility defined by $R_{2}$ especially for small scaled return intervals: the curves for all thresholds for $\tau_{0}$ in the smallest subset and biggest subset approximately collapse onto two separate solid curves. $P_{q}\left(\tau \mid \tau_{0}\right)$ shows bigger probabilities for $\tau_{0}$ in the smallest (biggest) subset when $\tau /\langle\tau\rangle$ is small (big), and this indicates that small (big) intervals $\tau_{0}$ tend to be followed by small (big) intervals $\tau$. This can be regarded as a proof of the existence of short-term memory in realized volatility return intervals, while all the $P_{q}\left(\tau \mid \tau_{0}\right)$ curves for the shuffled data collapse to a single exponential curve (not shown).

\subsection{Long memory in realized volatility and its return intervals}

To further investigate the long-term memory of the realized volatility and its return intervals we use the detrended fluctuation analysis (DFA) method [44, 45, 46, 47, 48], known as a general method of examining the long-term correlation in time series analysis. The DFA method computes the average fluctuation $F(l)$ of the cumulative series $y(t)=\sum_{t^{\prime}=1}^{t} x\left(t^{\prime}\right)$ of data series $x\left(t^{\prime}\right)$ as

$$
F(l)=\frac{1}{N_{l}} \sum_{i}^{N_{l}} \sum_{t=1}^{l}(y(t)-\tilde{y}(t))^{2}
$$

where $N_{l}$ is the number of windows with fixed $l$ data points, and $\tilde{y}(t)$ is a local linear estimation for $y(t)$ in a certain window $i$. It is expected that $F(l)$ scales with $l$ as

$$
F(l) \sim l^{\alpha},
$$

The DFA method provides an accurate estimation of long-range correlation which do not depend on the length of the time series, and the scaling exponent $\alpha$ is supposed to be equal to the Hurst exponent when $\alpha \leq 1$ [49]. Generally, for $\alpha>0.5$ the time series are long-term correlated, and for $\alpha=0.5$ the time series are uncorrelated.

Fig. 5 presents the detrended fluctuation functions $F(l)$ of the realized volatility for SSEC and stock 600028. A crossover behavior is observed: for small scales of $l, F(l)$ obeys a power law with a relatively small exponent; while for large scales of $l, F(l)$ obeys a power law with a relatively large exponent. The exponent $\alpha$ is estimated to be 

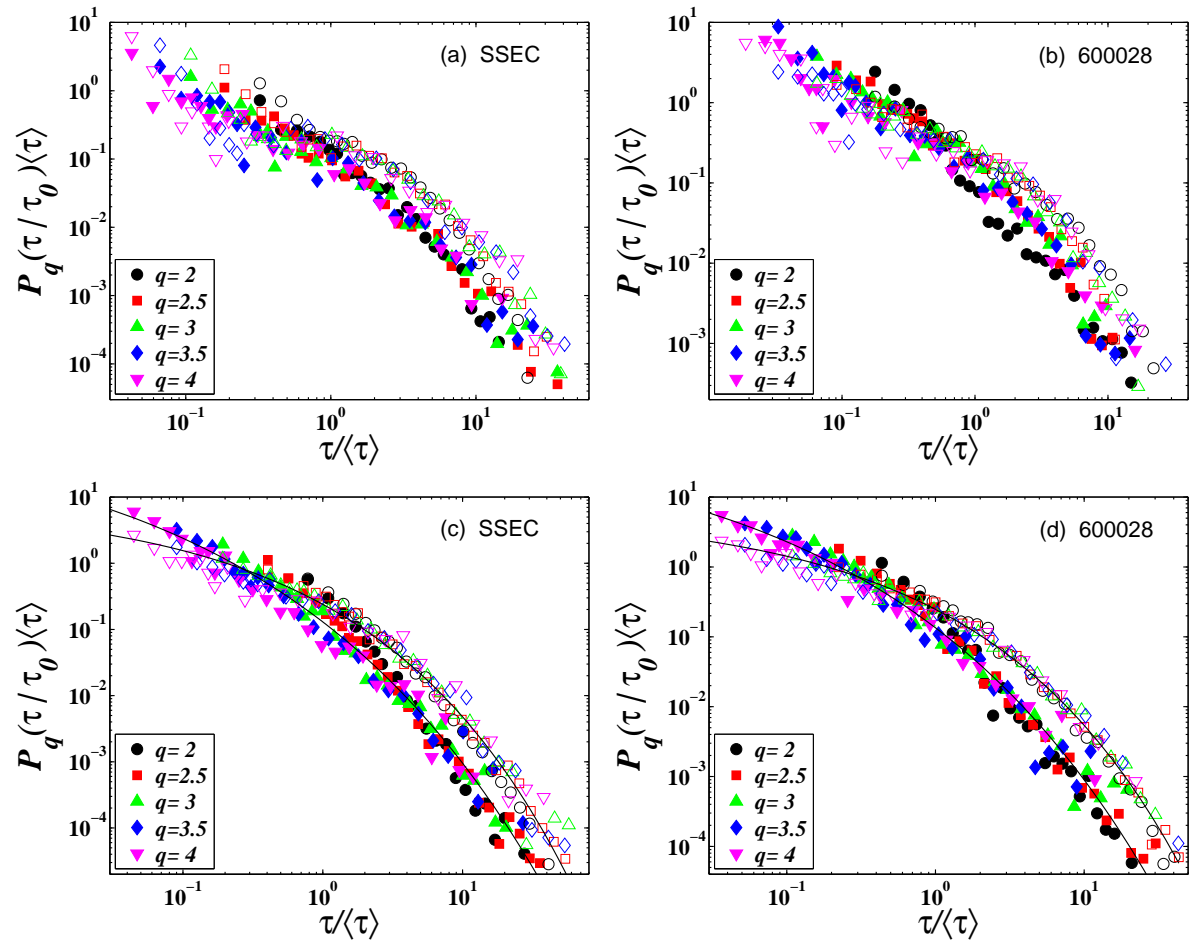

Figure 4: (Color online) Conditional PDFs of the scaled return intervals in the smallest 1/4 subset (filled symbols) and the largest $1 / 4$ subset (open symbols) for volatilities defined by (a) $R_{1}$ for SSEC, (b) $R_{1}$ for stock 600028 , (c) $R_{2}$ for SSEC, and (d) $R_{2}$ for stock 600028. The Solid lines in (c) and (d) are the stretched exponential fits.

$0.68 \pm 0.01$ and $0.86 \pm 0.02$ for SSEC and $0.69 \pm 0.01$ and $0.98 \pm 0.03$ for stock 600028 respectively in small scale and large scale regions, apparently larger than 0.5 . Similar results are observed for other constituent stocks. Therefore, we can conclude that the realized volatilities are long-term correlated.

We also compute the detrended fluctuation functions $F(l)$ of the return intervals of realized volatility as shown in Fig. 5, and observe similar crossover behavior. The estimation of the crossover point which separates the two power-law regions becomes an important task, since it essentially affects the determination of exponent $\alpha$. A simple least squares estimation method is applied to determine the value of the threshold by minimizing the square distance between $F(l)$ and its best power-law fits in small scale and large scale regions. In fact we have used the same least squares estimation method to find out the crossover point for $F(l)$ of the realized volatility. In Fig. 5 (a) and (b), the solid lines are power-law fits in small scale and large scale regions, respectively. Apparently the crossover tends to appear at smaller scales when the threshold $q$ increases as quantitatively illustrated in Fig. 6 (a).

As with this least squares estimation method, we can further test the relation between the exponent $\alpha$ of the return intervals and the threshold $q$, and see how the long-term memory of the return intervals varies with the change of $q$. In Fig. 6(b) and (c), the exponent $\alpha$ for SSEC and stock 600028 are plotted as a function of the threshold $q$. The curves fluctuate a little for relatively large $q$ due to the poor statistics of reduced interval samples. In general, the Hurst exponents in both regions for small scales and large scales show decreasing tendencies when $q$ increases. Though the long-term correlation of the return intervals is weakened with the increase of $q$, the exponent $\alpha$ for all thresholds is apparently larger than 0.5 . For the shuffled realized volatility data, the exponent $\alpha$ of the return intervals displays a value close to 0.5 . This indicates that the long-term memory of the return intervals may arise from the long-term memory of original volatility records. 

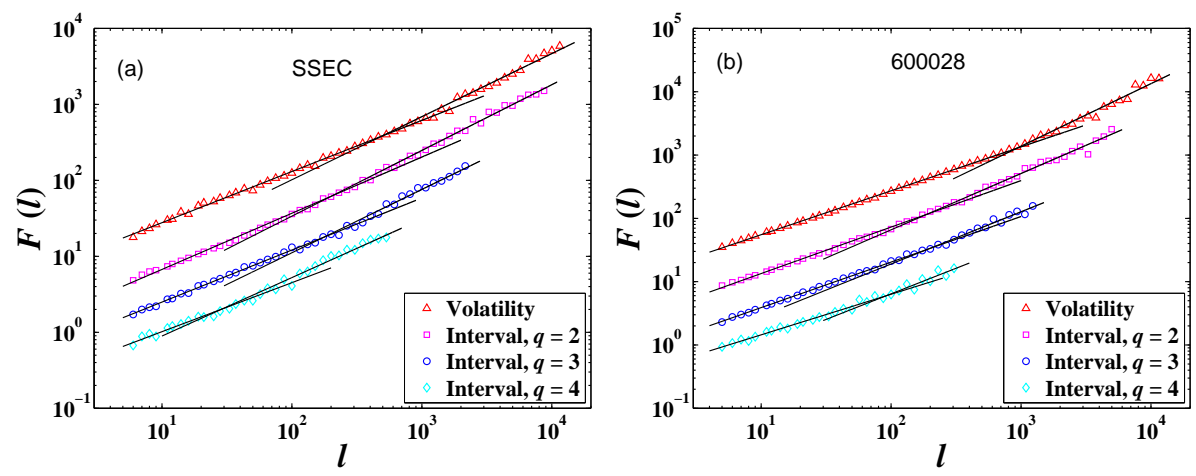

Figure 5: (Color online) Detrended fluctuation functions $F(l)$ of the realized volatility return intervals and the original realized volatilities for (a) SSEC and (b) stock 600028. The curves are vertically shifted for clarity.
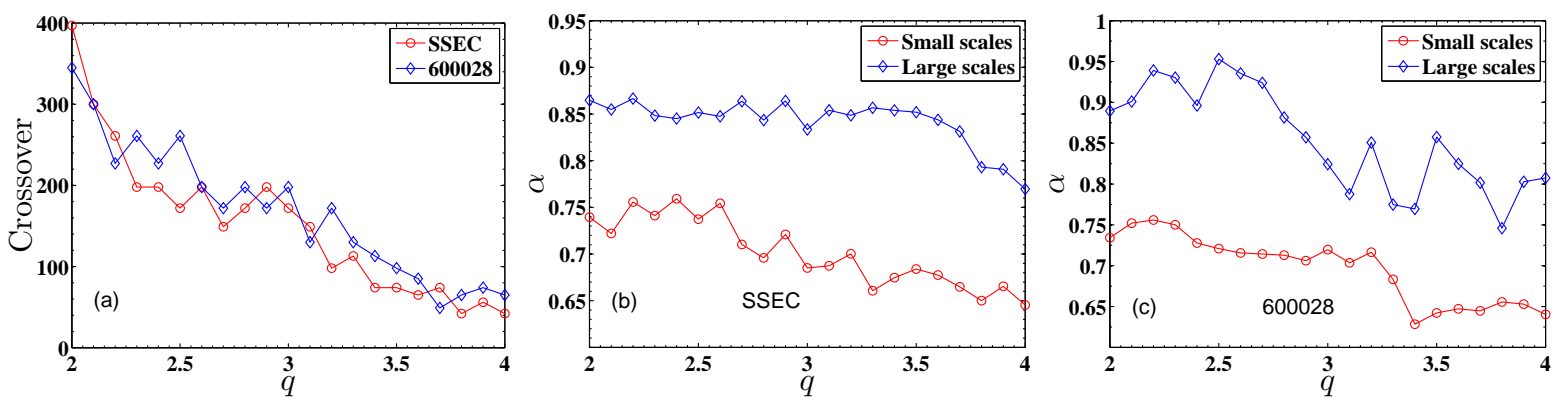

Figure 6: (Color online) (a) Crossover point as a function of $q$ for SSEC (circles) and stock 600028 (diamonds). Exponent $\alpha$ of the realized volatility return intervals for large scales (diamonds) and small scales (circles) for (b) SSEC and (c) stock 600028.

\section{Conclusion}

In summary, we have studied the statistical properties of the return intervals of 1-min realized volatility based on the high-frequency intraday data for the SSEC index and 22 liquid constituent stocks. The Kolmogorov-Smirnov test shows that 20 stocks (out of 22 stocks) and the SSEC exhibit scaling behaviors. We found that the scaling behavior of the return interval distribution of the realized volatility is significantly improved compared with that of the ordinary volatility defined by the closest tick prices to the minute marks. We further adopted the KS goodnessof-fit test using the $K S$ and weighted $K S W$ statistics to study the particular form of the scaling distribution, and found the scaling function for 8 constituent stocks can be well-approximated by a stretched exponential distribution $f(\tau /\langle\tau\rangle)=c e^{-a(\tau /\langle\tau\rangle)^{\gamma}}$. We calculated the relation between the exponent $\gamma$ estimated from the stretched exponential fit of $P_{q}(\tau)$ and the threshold $q$, and further demonstrated the improved scaling behavior of the realized volatility. The similarity of $P_{q}(\tau)$ for different stocks is also observed for the realized volatility.

We then investigated the memory effect of the realized volatility return intervals for the SSEC and 22 constituent stocks. Short-term memory is revealed by the observation of the conditional probability distribution $P_{q}\left(\tau \mid \tau_{0}\right)$ which also shows good scaling behavior for the realized volatility. Using the DFA method, we found that long-term memory exists in both realized volatility and its return intervals, and the exponent $\alpha$ of the realized volatility return intervals shows a decreasing tendency with the increase of the threshold $q$.

\section{Acknowledgments:}

This work was partially supported by the Shanghai Educational Development Foundation (2008CG37 and 2008SG29), the National Natural Science Foundation of China (70501011), and the Program for New Century Excellent Talents in University (NCET-07-0288). 


\section{References}

[1] A. Bunde, J. F. Eichner, S. Havlin, J. W. Kantelhardt, The effect of long-term correlations on the return periods of rare events, Physica A 330 (2003) $1-7$

[2] A. Bunde, J. F. Eichner, S. Havlin, J. W. Kantelhardt, Return intervals of rare events in records with long-term persistence, Physica A 342 (2004) 308-314.

[3] A. Bunde, J. F. Eichner, J. W. Kantelhardt, S. Havlin, Long-term memory: A natural mechanism for the clustering of extreme events and anomalous residual times in climate records, Phys. Rev. Lett. 94 (2005) 048701.

[4] P. Bak, K. Christensen, L. Danon, T. Scanlon, Unified scaling law for earthquakes, Phys. Rev. Lett. 88 (2002) 178501.

[5] A. Corral, Long-term clustering, scaling, and universality in the temporal occurrence of earthquakes, Phys. Rev. Lett. 92 (2004) 108501.

[6] A. Saichev, D. Sornette, "Universal" distribution of interearthquake times explained, Phys. Rev. Lett. 97 (2006) 078501.

[7] C. Pennetta, Distribution of return intervals of extreme events, Eur. Phys. J. B 50 (2006) 95-98.

[8] P. Olla, Return times for stochastic processes with power-law scaling, Phys. Rev. E 76 (2007) 011122.

[9] J. F. Eichner, J. W. Kantelhardt, A. Bunde, S. Havlin, Extreme value statistics in records with long-term persistence, Phys. Rev. E 73 (2006) 016130.

[10] J. F. Eichner, J. W. Kantelhardt, A. Bunde, S. Havlin, Statistics of return intervals in long-term correlated records, Phys. Rev. E 75 (2007) 011128.

[11] M. I. Bogachev, J. F. Eichner, A. Bunde, Effect of nonlinear correlations on the statistics of return intervals in multifractal data sets, Phys. Rev. Lett. 99 (2007) 240601.

[12] F. Mainardi, M. Raberto, R. Gorenflo, E. Scalas, Fractional calculus and continuous-time finance II: The waiting-time distribution, Physica A 287 (2000) 468-481.

[13] L. Sabatelli, S. Keating, J. Dudley, P. Richmond, Waiting time distributions in financial markets, Eur. Phys. J. B 27 (2002) $273-275$.

[14] P. C. Ivanov, A. Yuen, B. Podobnik, Y.-K. Lee, Common scaling patterns in intertrade times of U. S. stocks, Phys. Rev. E 69 (2004) 056107.

[15] E. Scalas, R. Gorenflo, H. Luckock, F. Mainardi, M. Mantelli, M. Raberto, Anomalous waiting times in high-frequency financial data, Quant. Financ. 4 (2004) 695-702.

[16] E. Scalas, T. Kaizoji, M. Kirchler, J. Huber, A. Tedeschi, Waiting times between orders and trades in double-auction markets, Physica A 366 (2006) 463-471.

[17] Z.-Q. Jiang, W. Chen, W.-X. Zhou, Scaling in the distribution of intertrade durations of Chinese stocks, Physica A 387 (2008) $5818-5825$.

[18] K. Yamasaki, L. Muchnik, S. Havlin, A. Bunde, H. E. Stanley, Scaling and memory in volatility return intervals in financial markets, Proc. Natl. Acad. Sci. U.S.A. 102 (2005) 9424-9428.

[19] F.-Z. Wang, K. Yamasaki, S. Havlin, H. E. Stanley, Scaling and memory of intraday volatility return intervals in stock markets, Phys. Rev. E 73 (2006) 026117.

[20] F. Wang, P. Weber, K. Yamasaki, S. Havlin, H. E. Stanley, Statistical regularities in the return intervals of volatility, Eur. Phys. J. B 55 (2007) 123-133.

[21] I. Vodenska-Chitkushev, F.-Z. Wang, P. Weber, K. Yamasaki, S. Havlin, H. E. Stanley, Comparison between volatility return intervals of the S\&P 500 index and two common models, Eur. Phys. J. B 61 (2008) 217-223.

[22] W.-S. Jung, F.-Z. Wang, S. Havlin, T. Kaizoji, H. T. Moon, H. E. Stanley, Volatility return intervals analysis of the Japanese market, Eur. Phys. J. B 62 (2008) 113-119.

[23] T. Qiu, L. Guo, G. Chen, Scaling and memory effect in volatility return interval of the Chinese stock market, Physica A 387 (2008) $6812-6818$.

[24] J. W. Lee, K. E. Lee, P. A. Rikvold, Waiting-time distribution for Korean stock-market index KOSPI, J. Korean Phys. Soc. 48 (2006) S123S126.

[25] F.-Z. Wang, K. Yamasaki, S. Havlin, H. E. Stanley, Indication of multiscaling in the volatility return intervals of stock markets, Phys. Rev. E 77 (2008) 016109.

[26] F.-Z. Wang, K. Yamasaki, S. Havlin, H. E. Stanley, Multifactor analysis of multiscaling in volatility return intervals, Phys. Rev. E 79 (2009) 016103.

[27] T. Kaizoji, M. Kaizoji, Power law for the ensemble of stock prices, Physica A 344 (2004) 240-243.

[28] F. Ren, W.-X. Zhou, Multiscaling behavior in the volatility return intervals of Chinese indices, EPL 84 (2008) 68001.

[29] F. Ren, L. Guo, W.-X. Zhou, Statistical properties of volatility return intervals of Chinese stocks, Physica A 388 (2009) 881-890.

[30] T. G. Andersen, T. Bollerslev, F. X. Diebold, P. Labys, The distribution of realized exchange rate volatility, J. Am. Stat. Assoc. 96 (453) (2001) 42-55

[31] T. G. Andersen, T. Bollerslev, F. X. Diebold, P. Labys, The distribution of realized stock return volatility, J. Financ. Econ. 61 (2001) $43-76$.

[32] G. W. Schwert, Stock market volatility, Financ. Analysts J. 46 (1990) 23-34.

[33] D. Hsieh, Chaos and non-linear dynamics: Application to financial markets, J. Financ. 5 (1991) $1839-1877$.

[34] W. J. den Haan, A. Levin, A practitioner's guide to robust covariance matrix estimation, NBER Working Paper No. T0197 (1996).

[35] B. Bollen, B. Inder, Estimating daily volatility in financial markets utilizing intraday data, J. Emp. Financ. 9 (2002) 551-562.

[36] T. G. Andersen, T. Bollerslev, F. X. Diebold, Parametric and nonparametric volatility measurement, in: Y. Ait-Sahalia, L. P. Hansen (Eds.), Handbook of Financial Econometrics, Elsevier, Amsterdam, 2009.

[37] X.-H. Ni, W.-X. Zhou, Intraday pattern in bid-ask spreads and its power-law relaxation for Chinese A-share stocks, J. Korean Phys. Soc. 54 (2009) 786-791.

[38] D. A. Darling, The Kolmogorov-Smirnov, Cramer-von Mises tests, Ann. Math. Stat. 28 (1957) $823-838$.

[39] M. A. Stephens, EDF statistics for goodness of fit and some comparisons, J. Am. Stat. Assoc. 69 (1974) $730-737$.

[40] N. V. Smirnov, Table for estimating the goodness of fit of empirical distributions, Ann. Math. Stat. 19 (1948) $279-281$.

[41] I. T. Young, Proof without prejudice: Use of the Kolmogorov-Smirnov test for the analysis of histograms from flow systems and other sources, J. Histochem. Cytochem. 25 (1977) 935-941.

[42] A. Clauset, C. R. Shalizi, M. E. J. Newman, Power-law distributions in empirical data, SIAM Rev. 48 (2009) in press. 
[43] M. C. Gonzalez, C. A. Hidalgo, A.-L. Barabási, Understanding individual human mobility patterns, Nature 453 (2008) 779-782.

[44] C.-K. Peng, S. V. Buldyrev, S. Havlin, M. Simons, H. E. Stanley, A. L. Goldberger, Mosaic organization of DNA nucleotides, Phys. Rev. E 49 (1994) 1685-1689.

[45] K. Hu, P. C. Ivanov, Z. Chen, P. Carpena, H. E. Stanley, Effect of trends on detrended fluctuation analysis, Phys. Rev. E 64 (2001) 011114.

[46] J. W. Kantelhardt, E. Koscielny-Bunde, H. H. A. Rego, S. Havlin, A. Bunde, Detecting long-range correlations with detrended fluctuation analysis, Physica A 295 (2001) 441-454.

[47] Z. Chen, P. C. Ivanov, K. Hu, H. E. Stanley, Effect of nonstationarities on detrended fluctuation analysis, Phys. Rev. E 65 (2002) 041107.

[48] Z. Chen, K. Hu, P. Carpena, P. Bernaola-Galvan, H. E. Stanley, P. C. Ivanov, Effect of nonlinear filters on detrended fluctuation analysis, Phys. Rev. E 71 (2005) 011104

[49] A. V. Coronado, P. Carpena, Size effects on correlation measures, J. Biol. Phys. 31 (2005) 121-133. 